Journal of Attention Disorders Vol. 11(1):37-48 (2007)

ISSN: $1087-0547$

doi: $10.1177 / 1087054706295664$

This is a peer reviewed pre-print version of the following article: Child and Parent Predictors of Perceptions of ParentChild Relationship Quality, which has been published in final form at:

http://www.sagepub.com/home.nav

http://jad.sagepub.com/

http://jad.sagepub.com/content/11/1/37.full.pdf + html

(C) 2007 Sage Publications Ltd.

\title{
Child and Parent Predictors of Perceptions of Parent-Child Relationship Quality
}

\author{
Alyson C. Gerdes, Marquette University, Betsy Hoza, University of Vermont, L. \\ Eugene Arnold, Ohio State University, Stephen P. Hinshaw, University of California, \\ Berkeley, Karen C. Wells, Duke University Medical Center, Lily Hechtman, \\ Montreal Children's Hospital/McGill University, Laurence L. Greenhill, New York \\ State Psychiatric Institute/Columbia University, James M. Swanson, University of \\ California, Irvine, William E. Pelham, State University of New York, Buffalo, \\ Timothy Wigal, University of California, Irvine
}

Objective/Method:

Predictors of perceptions of parent-child relationship quality were examined for 175 children with ADHD, 119 comparison children, and parents of these children, drawn from the follow-up phase of the Multimodal Treatment Study of Children with ADHD.

Results/Conclusion:

Children with ADHD perceived their mothers and fathers as more power assertive than comparison children. Children higher on depressive symptomatology also perceived their mothers and fathers as less warm and more power assertive. Mothers perceived themselves as more power assertive and fathers perceived themselves as less warm if they were higher on depressive symptomatology themselves or had children with ADHD or higher levels of depressive symptomatology. Several interactions indicated that the association between child factors and parental perceptions of warmth and power assertion often depended on parental depressive symptomatology. The findings resolve a previous contradiction in the literature regarding the relationship between child depressive symptoms and parental perceptions of parent-child relationship quality.

Given the importance of parent-child relationships in child development and adjustment (Paulson, Hill, \& Holmbeck, 1991; Herman \& McHale, 1993), perceptions of parent-child relationship quality may be particularly important to examine in at-risk populations that are prone to more negative parent-child relationships. Children with ADHD and their parents engage in more negative behaviors and fewer positive behaviors during interactions with each other compared to comparison children and their parents (e.g., Buhrmester, Camparo, Christensen, Gonzalez, \& Hinshaw, 1992; Campbell, March, Pierce, Ewing, \& Szumowski, 1991; Johnston, 1996). As reported by Gerdes, Hoza, and Pelham (2003), the accumulation of these negative interactions over time likely negatively affects the overall quality of the parent-child relationship; however, with few exceptions, the literature has not examined factors that may contribute to child and parental perceptions of relationship quality in this population.

One exception is a study conducted by Gerdes et al. (2003) examining the role of child ADHD status (i.e., whether a child had ADHD), child depressive symptomatology, and their interaction in predicting child and parental perceptions of parent-child relationship quality. The 
findings suggested that factors predicting relationship perceptions differed for parents and children. Unfortunately, this study only examined boys and did not explore the role that parental depressive symptomatology may play in parental and child perceptions of relationship quality. Therefore, the goal of the present study was to extend the findings by Gerdes et al. by examining factors that may similarly or differently contribute to child and parental perceptions of relationship quality by simultaneously examining the relative and combined predictive power of child ADHD status, child depressive symptomatology, parental depressive symptomatology, and their interactions.

A growing body of literature suggests that children with ADHD exhibit poor social acuity in the peer domain (i.e., they exhibit a positive bias in their perceptions of their peer relationships compared to comparison children). Relative to comparison children, children with ADHD report perceiving that an unfamiliar child enjoyed playing with them more and liked them more relative to the other child's report (Diener \& Milich, 1997). In addition, despite evidence that children with ADHD demonstrate less socially effective behavior than comparison children while interacting with a child confederate, children with ADHD provide more positive performance evaluations for these peer interactions and report greater liking of the child confederate relative to comparison children, especially following failure (Hoza, Waschbusch, Pelham, Molina, \& Milich, 2000). Furthermore, children with ADHD do not differ from comparison children in their reports of having friends, even though their peers and mothers rate them as less socially accepted (Hinshaw \& Melnick, 1995). Finally, children with ADHD overestimate their social acceptance relative to both maternal and teacher reports more than comparison children do (Hoza et al., 2004; Hoza, Pelham, Dobbs, Owens, \& Pillow, 2002). Gerdes et al. (2003) found initial support that this positive bias also extends to perceptions of parent-child relationships. Parents of children with ADHD reported more negative perceptions of relationship quality than parents of comparison children; however, no difference in relationship perceptions was found between ADHD and comparison children. Based on this previous work, it was expected that child ADHD status would predict more negative parental perceptions, but would not be a significant predictor of child perceptions of relationship quality.

In addition, it was expected that child depressive symptomatology would predict less positive child perceptions of the parent-child relationship; however, due to somewhat conflicting findings, it was unclear if child depressive symptomatology would be a significant predictor of parental perceptions. Research examining children with depressive symptomatology suggests that they have self-derogatory perceptions; they perceive themselves as less competent in a variety of areas (e.g., social, athletic, and global self-worth) relative to their nondepressed peers (Asarnow \& Bates, 1988; Epkins, 1996). In their longitudinal work, McGrath and Repetti (2002) also found that child depressive symptomatology was a significant predictor of changes in self-perceptions over time; more depressive symptomatology was associated with more negative perceptions of scholastic competence, social acceptance, and global self-worth. Given this research, together with the finding by Gerdes et al. (2003) that child depressive symptomatology was a significant predictor of negative child perceptions of relationship quality, we expected to find a similar association in the current study. However, whether child depressive symptomatology also would predict parental perceptions of relationship quality was less clear. Although research has found an association between parent-perceived warmth and power assertion and child internalizing problems (O'Brien \& Bahadur, 1998), Gerdes and colleagues did not find child depressive symptomatology to be a significant predictor of parental perceptions of relationship quality.

On the other hand, it was expected that parental depressive symptomatology would predict 
less positive perceptions of relationship quality for both parents and children. Downey and Coyne (1990) provide a comprehensive review of the association between parental depression and parenting behavior. Their review shows that, compared to their nondepressed counterparts, parents with high levels of depressive symptoms respond less positively to their children, exhibit less positive affect, display more irritable and hostile behavior directed toward their children, and are more likely to use coercion to manage child behavior. A more recent study examining parental warmth and control provides further support for the positive association between parental depressive symptomatology and negative parenting behavior. Cummings, Keller, and Davies (2005) found that parental depressive symptomatology is associated with more parental control and less warmth. Given the impact that parental depressive symptomatology has on parenting behavior, we expected parental depressive symptomatology to predict more negative parent and child perceptions of relationship quality. Finally, several interactions were examined (ADHD $\times$ Child Depressive Symptomatology, ADHD $\times$ Parent Depressive Symptomatology, and Child Depressive Symptomatology $\times$ Parent Depressive Symptomatology); however, due to a lack of prior research in this area, specific predictions related to the interactions were not made.

\section{Method}

\section{Participants}

In all, 175 boys and girls with ADHD, 119 local normative comparison group (LNCG) boys and girls, and their parents, drawn from the follow-up phase of the Multimodal Treatment Study of Children with ADHD (MTA), served as participants. Children with ADHD met criteria for ADHD, Combined Type at the baseline assessment, were between the ages of 7.0 and 9.9 years at the beginning of the study, and had either undergone a 14-month treatment protocol administered by MTA staff or had been part of a community care group who chose their own treatment(s). Children with ADHD treated by MTA staff received medication management, behavior therapy, or combined treatment (medication management and behavior therapy). A detailed description of these treatments may be found elsewhere (Arnold et al., 1997; Greenhill et al., 1996; Wells et al., 2000). Approximately two thirds of families who were assigned to choose their own community treatment received medication for their children (MTA Cooperative Group, 1999).

The measures used in the present study were administered at the 24-month assessment, 10 months after the end of treatment for most families. The LNCG children were recruited at this time. These children were recruited from the same schools as the children with ADHD in a stratified manner to represent the same proportions by sex and current grade of the children with ADHD. The school either directly sent letters to families of all children in the appropriate grade and of the appropriate sex or randomly selected a child of the appropriate grade and sex and contacted the family for the site. The goal of a minimum 50\% participation rate was achieved. LNCG children were not screened for any psychological problems, including ADHD; however, they had to pass the same exclusion criteria as the children with ADHD (e.g., if the child's IQ was below 80 he or she was excluded).

\section{Measures}

Parent-Child Relationship Questionnaire (PCRQ; Furman \& Giberson, 1995).

The short version of the PCRQ consists of 40 items that make up five subscales; the warmth and power assertion subscales were the only two used in the present study. Both a child and parent version of the PCRQ exist; children and parents completed the appropriate version 
about their relationships with each other. Each question is answered on a scale ranging from hardly at all (1) to extremely much (5). A sample item from the warmth subscale is: "How much do you and your mother love each other?" A sample item from the power assertion subscale is: "How much does your mother spank you when you misbehave?" Using Cronbach's alpha, acceptable internal consistencies were obtained for these subscales in the current sample (range $=.83$ to .84 for children's reports about their mothers, range $=.84$ to .85 for mothers' reports about their children, range $=.88$ to .91 for children's reports about their fathers, and range $=.85$ to .88 for fathers' reports about their children).

Children's Depression Inventory (CDI; Kovacs, 1992).

The CDI is a widely used self-report measure of child depressive symptomatology. It is made up of 27 items, each with three response choices. For example, I feel like crying every day (2), I feel like crying many days (1), I feel like crying once in a while (0). Higher scores represent greater depressive symptomatology. An extensive body of literature supports the CDI as a reliable and valid measure of child depressive symptoms (Kovacs, 1992).

Beck Depression Inventory (BDI; Beck, Rush, Shaw, \& Emery, 1979).

The BDI is a widely used self-report measure of adult depressive symptomatology. It is composed of 21 items, each with four response choices. For example, I do not feel sad (0), I feel sad much of the time (1), I am sad all the time (2), I am so sad or unhappy that I can't stand it (3). Similar to the CDI, higher scores represent greater depressive symptomatology. The BDI has been shown to be a reliable and valid self-report measure of adult depressive symptoms (Beck, Steer, \& Garbin, 1988).

\section{Results}

\section{Preliminary Analyses}

Comparisons of children with ADHD by treatment group on dependent variables.

Our main goal was to examine the predictive power of child ADHD status, child depressive symptomatology, parental depressive symptomatology, and their interactions on perceptions of parent-child relationship quality; however, before conducting the primary analyses, a MANOVA approach was used to examine whether our ADHD sample differed on our dependent variables based on the treatment they received. Because no significant multivariate effects for treatment group emerged, children with ADHD were collapsed across treatment group for future analyses.

Comparisons of children with ADHD and LNCG children on demographic variables.

Using ANOVAs for continuous variables and chi-square for categorical variables, the ADHD and LNCG groups were compared on several demographic variables, including age, gender, ethnicity, parents' educational level, income, marital status, and family composition, to confirm comparability of the groups. As Table 1 indicates, none of the demographic comparisons was significant; however, as expected, the LNCG group had significantly lower ratings of parent-and teacher-reported inattention, hyperactivity, impulsivity, and oppositionality symptoms on the Swanson, Nolan, and Pelham Version IV (SNAP; Swanson, 1992) than the ADHD group. These clinical differences confirmed that the LNCG children were an appropriate comparison group.

\section{Primary Analyses}


Using hierarchical regressions, we examined the ability of child ADHD status, child depressive symptomatology, parental depressive symptomatology, and their interactions to predict child and parental perceptions of parental warmth and power assertion. Eight hierarchical regressions were conducted: four predicting children's perceptions of their mothers' and fathers' warmth and power assertion and four predicting mothers' and fathers' perceptions of their own warmth and power assertion.

Predictors were centered prior to running the regression analyses. Continuous variables were centered by subtracting the mean from each variable; weighted effects coding was used to center child ADHD status (ADHD = .40, LNCG = -.60). At Step 1, child ADHD status (ADHD, LNCG) was entered. Child depressive symptomatology (based on the CDI) and parental depressive symptomatology (based on the BDI) were entered at Step 2. At Step 3, three 2-way interactions were entered (Child Depressive Symptomatology $\times$ Parental Depressive Symptomatology, ADHD $\times$ Child Depressive Symptomatology, and ADHD $\times$ Parental Depressive Symptomatology). Finally, the 3-way interaction (ADHD $\times$ Child Depressive Symptomatology $\times$ Parental Depressive Symptomatology) was entered at Step 4. Interpretation of betas was made at the third step, because this was the last step at which significant predictors entered the equation. Individual interaction terms only were examined if the interaction block was significant at $p<.05$. Significant interactions were decomposed using a method described by Aiken and West (1991).

Children's perceptions of their relationships with their mothers.

Child ADHD status significantly predicted children's perceptions of their mothers' power assertion, accounting for $2 \%$ of the variance. As shown in Table 2, examination of the betas indicated that children with ADHD perceived their mothers as more power assertive than LNCG children did. The depression block (child and maternal depressive symptomatology) also was a significant predictor of children's perceptions of their mothers' warmth and power assertion, accounting for $5 \%$ and $7 \%$ of

\begin{tabular}{llll}
\hline & ADHD & LNCG & $p$ value \\
\hline Child Variables & & & \\
Age - Mean (SD) & $9.71(.80)$ & $9.91(1.12)$ & $n s$ \\
Male - Number (\%) & $137(78)$ & $97(82)$ & $n s$ \\
Ethnicity - Number (\%) & & & $n s$ \\
Caucasian & $157(90)$ & $101(85)$ & \\
African American & $14(8)$ & $8(7)$ & \\
Hispanic & $4(2)$ & $10(8)$ & $<.001$ \\
SNAP - Mother - Mean (SD) & & & $<.001$ \\
Inattention & $1.29(.68)$ & $.51(.54)$ & $<.001$ \\
Hyperactive & $.90(.68)$ & $.29(.44)$ & $<.001$ \\
Impulsivity & $1.07(.78)$ & $.34(.46)$ & $.36(.45)$ \\
ODD & $.91(.68)$ & & \\
SNAP - Teacher - Mean (SD) & & &
\end{tabular}




\begin{tabular}{llll} 
Inattention & $1.49(.81)$ & $.79(.80)$ & $<.001$ \\
Hyperactive & $.94(.76)$ & $.41(.57)$ & $<.001$ \\
Impulsivity & $.96(.83)$ & $.48(.72)$ & $<.001$ \\
ODD & $.71(.72)$ & $.32(.53)$ & $n s$ \\
Parent/Family Variables & & $n s$ \\
High School Graduate - Number (\%) & $168(96)$ & $115(97)$ & $n s$ \\
Mother & $163(93)$ & $113(95)$ & \\
Father & & & \\
Income - Number (\%) & $12(7)$ & $5(4)$ & $n s$ \\
\$0-\$20,000 & $68(39)$ & $33(28)$ & $n s$ \\
\$20,001-\$50,000 & $95(54)$ & $81(68)$ & \\
$>$ \$50,000 & $163(93)$ & $116(98)$ & \\
Married - Number (\%) & $169(97)$ & $117(98)$ & \\
Family Composition - Number (\%) & $6(3)$ & $2(2)$ & \\
2 Parents & & \\
1 Parent & & \\
\hline
\end{tabular}

Note: $\mathrm{LNCG}=$ local normative comparison group; SNAP = Swanson, Nolan, and Pelham Version IV; ODD $=$ Oppositional Defiant Disorder. ADHD $n=175, \mathrm{LNCG} n=119 . n s=$ not significant $a t p<.05$.

Table 1 Demographic Data 


\begin{tabular}{|c|c|c|c|c|c|c|c|c|c|c|c|c|}
\hline & \multicolumn{6}{|c|}{ Child Perceptions } & \multicolumn{6}{|c|}{ Mother Perceptions } \\
\hline & \multicolumn{3}{|c|}{ Warmth } & \multicolumn{3}{|c|}{ Power Assertion } & \multicolumn{3}{|c|}{ Warmth } & \multicolumn{3}{|c|}{ Power Assertion } \\
\hline & $\Delta R^{2}$ & $\Delta F$ & Beta & $\Delta R^{2}$ & $\Delta F$ & Beta & $\Delta R^{2}$ & $\Delta F$ & Beta & $\Delta R^{2}$ & $\Delta F$ & Beta \\
\hline Step 1 - ADHD status & .00 & 1.00 & .08 & .02 & $5.56^{*}$ & $.12 *$ & .08 & $24.93 * * *$ & $.24 * * *$ & .09 & $27.58 * * *$ & $.24 * * *$ \\
\hline Step 2 - depression block & .05 & $7.65^{* *}$ & & .07 & $11.91 * * *$ & & .06 & $10.63 * * *$ & & .09 & $14.83^{* * *}$ & \\
\hline Child dep & & & $-.23 * * *$ & & & $.29 * * *$ & & & $-.15^{* *}$ & & & $.15^{* *}$ \\
\hline Maternal dep & & & -.02 & & & -.05 & & & $-.21 * *$ & & & $.26 * * *$ \\
\hline Step 3 - two-way interactions & .02 & 2.18 & & .01 & .62 & & .03 & $3.80^{*}$ & & .01 & .74 & \\
\hline Child $\times$ Maternal Dep & & & .12 & & & -.03 & & & $.14^{*}$ & & & -.04 \\
\hline ADHD $\times$ Child Dep & & & -.06 & & & -.03 & & & .08 & & & -.06 \\
\hline ADHD $\times$ Maternal Dep & & & -.09 & & & .07 & & & $-.11^{\dagger}$ & & & .01 \\
\hline Total adjusted $R^{2}$ & .06 & & & .08 & & & .16 & & & .16 & & \\
\hline
\end{tabular}

Note: dep $=$ depressive symptomatology. Across all analyses, Step 4 predictors were never significant. Thus, all betas and adjusted $R^{2} s$ are from the third step of the equation. ADHD $n=175$; local normative comparison group $n=119 .{ }^{\dagger} p<.10 .{ }^{*} p<.05 .{ }^{* *} p<.01 .{ }^{* *} p<.001$.

Table 2 Child and Mother Perceptions of Maternal Warmth and Power Assertion 
the variance, respectively; children who reported more depressive symptoms saw their mothers as less warm and more power assertive.

Mothers' perceptions of their relationships with their children.

As shown in Table 2, child ADHD status and the depression block (child and maternal depressive symptomatology) were significant predictors of mothers' perceptions of their warmth and power assertion. ADHD status accounted for $8 \%$ and $9 \%$ of the variance in warmth and power assertion, respectively, whereas the depression block accounted for $6 \%$ and $9 \%$ of the variance in warmth and power assertion, respectively. Examination of the betas indicated that mothers of children with ADHD perceived themselves as less warm and more power assertive than mothers of LNCG children. In addition, mothers who reported a higher level of depressive symptomatology or had children who reported more depressive symptoms perceived themselves as less warm and more power assertive. However, the two-way interactions block also was a significant predictor of mothers' perceptions of warmth, accounting for 3\% of the variance. A significant Child Depressive Symptomatology $\times$ Maternal Depressive Symptomatology interaction indicated that the association between child depressive symptomatology and maternal perceptions of warmth depended on the level of maternal depressive symptomatology (see Figure 1). For mothers high on depressive symptomatology, the unstandardized regression coefficient indicated that there was not a significant association between child depressive symptomatology and maternal perceptions of warmth $(b=-.00, n s)$. On the other hand, for mothers low on depressive symptomatology, there was a significant negative association between child depressive symptomatology and maternal perceptions of warmth $(b=-.02, p<.001)$; higher levels of child depressive symptomatology were associated with mothers reporting less warmth. Finally, a marginally significant ADHD $\times$ Maternal Depressive Symptomatology interaction indicated that the association between ADHD status and maternal perceptions of warmth also depended on the level of maternal depressive symptomatology (see Figure 2). Specifically, there was a significant negative association between ADHD status and maternal perceptions of warmth for mothers high on depressive symptomatology $(b=-.36, p<.001)$; having a child with ADHD was associated with mothers perceiving themselves as less warm. However, there was not a significant association for mothers low on depressive symptomatology $(b=-.12, n s)$.

Children's perceptions of their relationships with their fathers.

Child ADHD status significantly predicted children's perceptions of their fathers' power assertion, accounting for 3\% of the variance. As shown in Table 3, examination of the betas indicated that children with ADHD perceived their fathers as more power assertive than LNCG children did. The depression block (child 


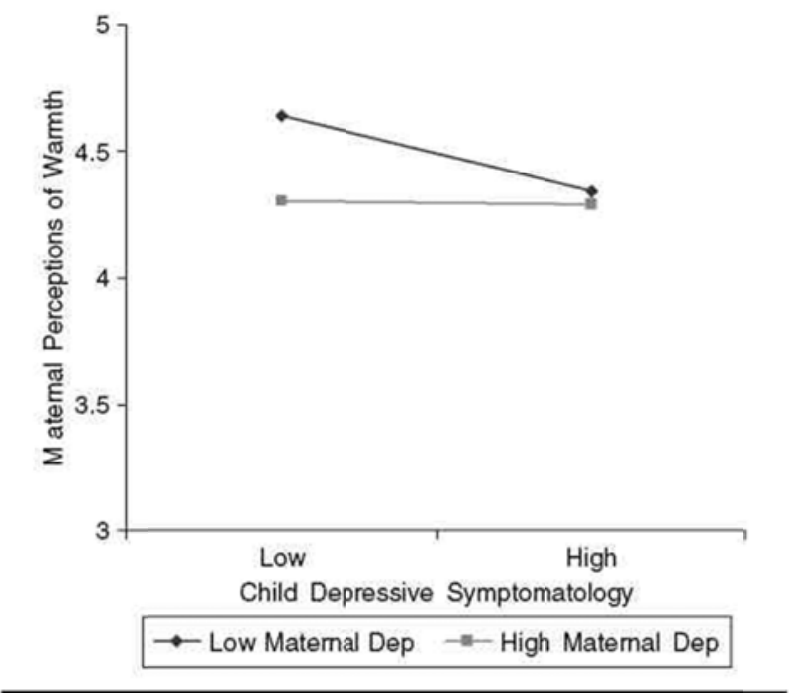

Note: Low and High on the $\mathrm{x}$ axis are one standard deviation below and above the mean, respectively, for child depressive symptoms.

Figure 1 Child Depressive Symptomatology $\times$ Maternal Depressive Symptomatology (Dep) Interaction Predicting Maternal Perceptions of Warmth

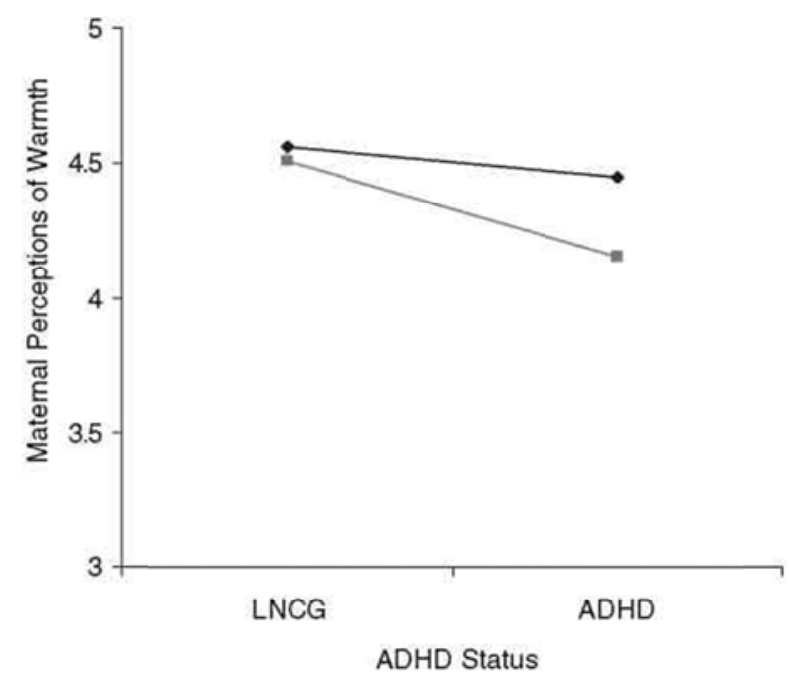

Low Maternal Dep $\rightarrow-$ High Maternal Dep

Figure 2 ADHD $\times$ Maternal Depressive Symptomatology (Dep) Interaction Predicting Maternal Perceptions of Warmth

and paternal depressive symptomatology) also was a significant predictor of children's perceptions of their fathers' warmth and power assertion, accounting for $16 \%$ and $10 \%$ of the 


\begin{tabular}{|c|c|c|c|c|c|c|c|c|c|c|c|c|}
\hline & \multicolumn{6}{|c|}{ Child Perceptions } & \multicolumn{6}{|c|}{ Father Perceptions } \\
\hline & \multicolumn{3}{|c|}{ Warmth } & \multicolumn{3}{|c|}{ Power Assertion } & \multicolumn{3}{|c|}{ Warmth } & \multicolumn{3}{|c|}{ Power Assertion } \\
\hline & $\Delta R^{2}$ & $\Delta F$ & Beta & $\Delta R^{2}$ & $\Delta F$ & Beta & $\Delta R^{2}$ & $\Delta F$ & Beta & $\Delta R^{2}$ & $\Delta F$ & Beta \\
\hline Step 1 - ADHD status & .00 & .38 & .08 & .03 & $9.79 * *$ & $.15^{* *}$ & .05 & $16.44 * * *$ & $-.18 * *$ & .08 & $24.16 * * *$ & $.21 * * *$ \\
\hline Step 2 - depression block & .16 & $28.22 * * *$ & & .10 & $17.28 * * *$ & & .08 & $12.47 * * *$ & & .05 & $8.28 * * *$ & \\
\hline Paternal dep & & & .05 & & & .03 & & & $-.13 *$ & & & $.20 * *$ \\
\hline Step 3 - two-way interactions & .02 & $2.75^{*}$ & & .01 & 1.44 & & .01 & 1.31 & & .05 & $5.50 * *$ & \\
\hline Child $\times$ Paternal Dep & & & .06 & & & .04 & & & .11 & & & $-.24 * * *$ \\
\hline ADHD $\times$ Child Dep & & & -.08 & & & -.05 & & & .06 & & & $-.11^{\dagger}$ \\
\hline ADHD $\times$ Paternal Dep & & & -.08 & & & .12 & & & -.03 & & & -.03 \\
\hline
\end{tabular}

Note: dep = depressive symptomatology. Across all analyses, Step 4 predictors were never significant. Thus, all betas and adjusted $R^{2} s$ are from the third step of the equation. ADHD $n=175$; local normative comparison group $n=119$.

${ }^{\dagger} p<.10 .{ }^{*} p<.05 . * * p<.01 . * * * p<.001$.

Table 3 Child and Father Perceptions of Paternal Warmth and Power Assertion 
variance, respectively; children who reported greater depressive symptomatology saw their fathers as less warm and more power assertive. Finally, the two-way interactions block was significant in predicting children's perceptions of their fathers' warmth; however, none of the betas was significant for the individual interactions.

Fathers' perceptions of their relationships with their children.

As shown in Table 3, child ADHD status was a significant predictor of fathers' perceptions of their warmth and power assertion, accounting for $5 \%$ and $8 \%$ of the variance, respectively. Examination of the betas indicated that compared to fathers of LNCG children, fathers of children with ADHD perceived themselves as less warm and more power assertive. The depression block (child and paternal depressive symptomatology) also significantly predicted fathers' perceptions of their warmth and power assertion, accounting for $8 \%$ and $5 \%$ of the variance, respectively; fathers who reported a higher level of depressive symptomatology or had children who reported more depressive symptoms perceived themselves as less warm and more power assertive. However, the two-way interactions block also was a significant predictor of fathers' perceptions of their power assertion, accounting for $5 \%$ of the variance. A significant Child Depressive Symptomatology $\times$ Paternal Depressive Symptomatology interaction indicated that the association between child depressive symptomatology and paternal perceptions of power assertion depended on the level of paternal depressive symptomatology (see Figure 3). For fathers high on depressive symptomatology, there was not a significant association between child depressive symptomatology and paternal perceptions of power assertion $(b=-.00, n s)$. On the other hand, for fathers low on depressive symptomatology, there was a significant positive association between child depressive symptomatology and paternal perceptions of power assertion $(b=.04, p<.001)$; higher levels of child depressive symptomatology were associated with fathers reporting more power assertion. Finally, a marginally significant ADHD $\times$ Child Depressive Symptomatology interaction indicated that the association between child depressive symptomatology and paternal perceptions of power assertion depended on whether or not the child also had ADHD (see Figure 4). Specifically, there was a significant positive association between child depressive symptomatology and paternal perceptions of power assertion for fathers of LNCG children $(b=$ $.03, p<.01)$; higher levels of child depressive symptomatology were associated with fathers reporting more power assertion. However, there was not a significant association for fathers of children with ADHD $(b=.01, n s)$.

\section{Supplementary Analyses}

To further examine the unexpected finding related to the role of child ADHD status in predicting child perceptions of parental power assertion, a set of paired $t$ tests were performed. $t$ tests were conducted comparing child perceptions of maternal and paternal power assertion to parental perceptions of maternal and paternal power assertion; these were conducted separately for our ADHD and LNCG group. Neither $t$ test was significant for the LNCG group, whereas both were significant for the ADHD group (maternal power assertion $t=-2.72$, 


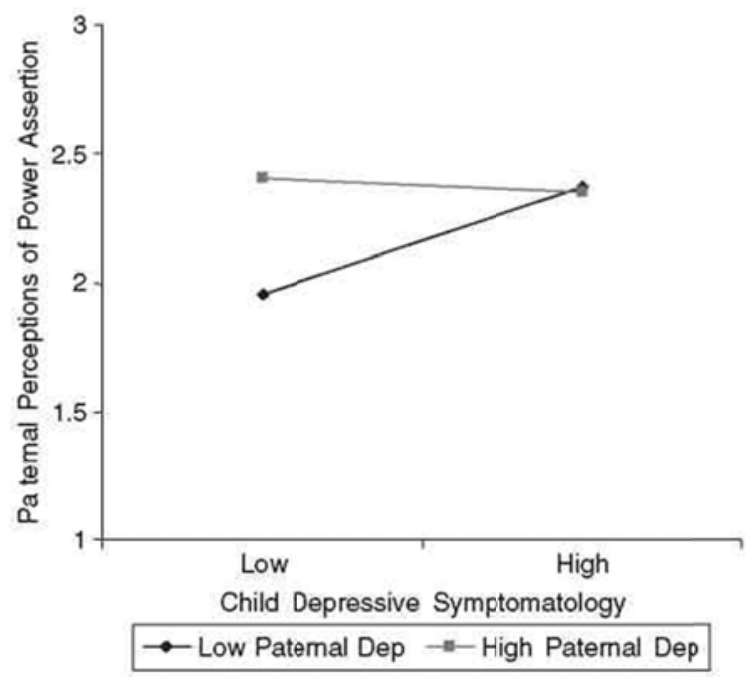

Note: Low and High on the $\mathrm{x}$ axis are one standard deviation below and above the mean, respectively, for child depressive symptoms.

Figure 3 Child Depressive Symptomatology $\times$ Paternal Depressive Symptomatology (Dep) Interaction Predicting Paternal Perceptions of Power Assertion

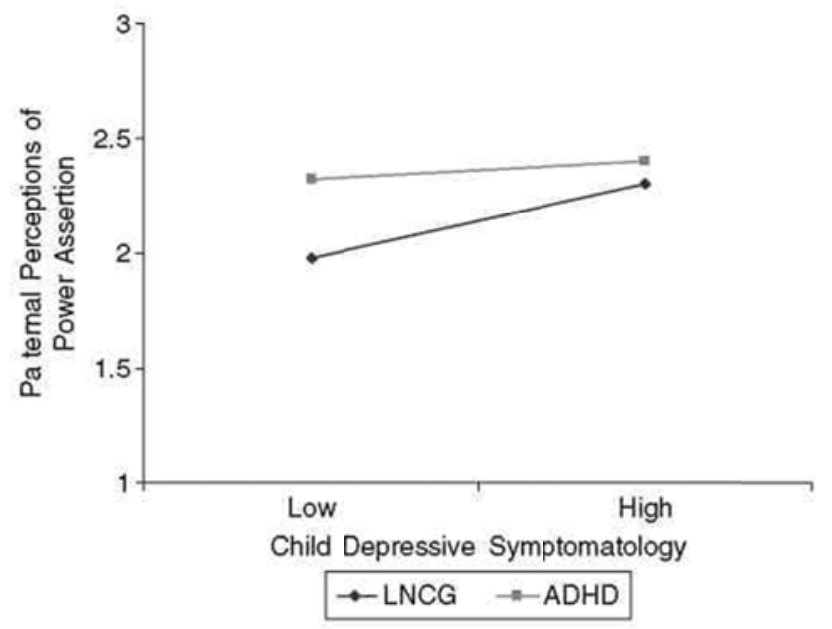

Note: Low and High on the $\mathrm{x}$ axis are one standard deviation below and above the mean, respectively, for child depressive symptoms.

Figure 4 ADHD $\times$ Child Depressive Symptomatology Interaction Predicting Paternal Perceptions of Power Assertion

$p<.01$ and paternal power assertion $t=-1.98, p<.05$ ), suggesting that children with ADHD reported less power assertion relative to their parents' reports.

\section{Discussion}

The goal of the present study was to further explore factors that may contribute to child and parental perceptions of relationship quality by simultaneously examining the relative and combined predictive power of child ADHD status, child depressive symptomatology, parental depressive symptomatology, and their interactions. Several interesting main effects and 
interactions emerged when examining child and parental perceptions of relationship quality. Findings are generally consistent with previous literature, but also add to the current literature by highlighting the role that parental depressive symptomatology plays in parental perceptions of the parent-child relationship. Furthermore, findings resolve a previous contradiction in the literature concerning the effects of child depressive symptoms on parental perceptions of relationship quality.

\section{Child and Parent Predictors of Child Perceptions of Parent-Child Relationship Quality}

Contrary to our prediction, child ADHD status significantly predicted child perceptions of maternal and paternal power assertion; children with ADHD actually perceived both their mothers and fathers as more power assertive (e.g., engaging in more yelling and spanking) than LNCG children did. This was unexpected because previous work demonstrated that children with ADHD exhibit poor social acuity, perceiving their interactions and relationships with peers as more positive than they really are (Diener \& Milich, 1997; Hinshaw \& Melnick, 1995; Hoza et al., 2000) and reporting greater social acceptance than is warranted (Hoza et al., 2004; Hoza et al., 2002). Although limited, research has shown that this positive illusory bias also is evident in children's perceptions of their parent-child relationships. When parent and child perceptions were directly compared on a dyad-by-dyad basis, Gerdes et al. (2003) found that children with ADHD reported more positive perceptions of their relationships with their parents relative to their parents' perceptions. Interestingly, follow-up analyses of the current data also indicate that when child and parental perceptions are directly compared, children with ADHD do report more positive perceptions of the relationship relative to their parents, whereas no difference is found between LNCG children and their parents. So although there is evidence that children with ADHD exhibit poor social acuity when reporting on the parent-child relationship, these positive illusions do not appear to be as strong as they are for perceptions of peer relationships. Recent work demonstrating that children with ADHD actually provide the most inflated ratings in their areas of greatest deficit (Hoza et al., 2004) may partially explain this. Unlike the peer relationships of children with ADHD, parent-child relationships are longer lasting and contain a member of the dyad (i.e., the parent) who is likely motivated to maintain a positive relationship. Given this, perhaps children's relationships with their parents are perceived as less of a source of deficit than their peer relationships, resulting in less need to inflate their perceptions. Differences in methodology also may explain why evidence appears stronger when examining peer relationships than when examining parent-child relationships.

As expected, child depressive symptomatology also was a significant predictor of child perceptions of their relationships with their parents. Children high on depressive symptomatology perceived both their mothers and fathers as less warm and more power assertive. This finding is both clinically intuitive (in that depressed individuals generally have a negative perceptual bias; Epkins, 1996) and also is consistent with previous work examining a similar population (Gerdes et al., 2003). What remains unanswered is whether child depressive symptomatology predicts poorer relationship quality because children with depressive symptomatology have more negative perceptions, or whether having a power assertive and less warm parent results in children experiencing more depressive symptoms. Both explanations are plausible and further investigation is needed to determine the direction of causation.

\section{Child and Parent Predictors of Parental Perceptions of Parent-Child Relationship Quality}

As expected, parental perceptions of relationship quality were predicted by child ADHD status. Mothers of children with ADHD reported more power assertion than mothers of LNCG 
children, and fathers of children with ADHD reported less warmth than fathers of LNCG children. This is not surprising given the large body of literature showing that the interactions between children with ADHD and their parents are characterized by more negativity and less positive behavior than those between comparison children and their parents (e.g., Buhrmester et al., 1992; Campbell et al., 1991; Johnston, 1996). This also is consistent with previous work (Gerdes et al., 2003) and with Patterson's coercion theory (Patterson, 1982). Similar to aggressive children and their parents, many children with ADHD and their parents seem to get caught in a negative coercive cycle, resulting in repeated negative behaviors being displayed by children and parents alike. These negative interactions likely take a toll on the overall quality of the parent-child relationship.

Additionally, parental and child depressive symptomatology significantly predicted both maternal and paternal perceptions of relationship quality. Mothers with higher levels of depressive symptomatology and mothers of children with higher levels of depressive symptoms reported greater power assertion, whereas fathers with higher levels of depressive symptomatology and fathers of children with higher levels of depressive symptoms reported less warmth. This is consistent with literature examining the association between parental depressive symptomatology and parenting behavior, which demonstrates that parents with high levels of depressive symptoms engage in less positive and more negative parenting behavior than parents who are not depressed (Cummings et al., 2005; Downey \& Coyne, 1990). Similar to our discussion related to the effects of child depressive symptomatology on self-perceptions, it is difficult to know if experiencing a high level of depressive symptoms results in more negative parental perceptions of relationship quality (either due to a negative cognitive bias or the negative effect of parental depression on parenting behavior) or if having a poor relationship with your child results in symptoms of depression.

It is interesting that child depressive symptomatology also was found to be associated with maternal reports of more power assertion and paternal reports of less warmth. Some studies have found an association between parent-perceived warmth and power assertion and child internalizing problems (O'Brien \& Bahadur, 1998), whereas other studies have not found child depressive symptomatology to be a significant predictor of parental perceptions of relationship quality (Gerdes et al., 2003). A possible explanation for this discrepancy is provided when discussing the interaction effects.

Perhaps the most interesting findings emerged when examining the interaction effects of parent and child factors on parental perceptions of relationship quality. Besides perceiving themselves as more power assertive than mothers of LNCG children, mothers of children with ADHD also perceived themselves as less warm, but only if they reported a high level of depressive symptomatology. This suggests that raising a child with ADHD alone is not associated with mothers perceiving themselves as less warm, but raising a child with ADHD, coupled with experiencing a high level of depressive symptoms, is associated with more negative perceptions of maternal warmth. This makes sense given what we know about the separate effects of parental depressive symptomatology (Cummings et al., 2005) and raising a child with ADHD (e.g., Buhrmester et al., 1992) on parenting behavior and suggests that depressed mothers who are raising a more challenging child may be particularly at-risk for the development of negative parent-child relationships.

In addition, child depressive symptomatology predicted maternal perceptions of warmth, but only for mothers who were low on depressive symptomatology. Similarly, child depressive symptomatology predicted paternal perceptions of power assertion, but only for fathers with low 
depressive symptomatology or fathers of LNCG children. These findings, together with the findings discussed earlier, suggest that one's own symptoms of depressive symptomatology may have a stronger influence on certain aspects of the parent-child relationship than the other relationship member's symptoms of psychopathology. In other words, if a parent is experiencing high levels of depressive symptoms themselves, their perceptions of the parent-child relationship are more negative, regardless of their child's level of depressive symptomatology. However, if a parent is not reporting depressive symptomatology, their child's level of depression becomes an important predictor of their perceptions. This suggests that parents are likely attuned to their children's moods, which affects their perceptions of certain aspects of the relationship, but not to the same extent that their own moods affect their perceptions of the relationship. This is consistent with the fact that to the extent that a depressed person "withdraws," he or she is less sensitive to the feelings and moods of others.

The current findings may help explain the conflicting findings regarding the association between child depressive symptomatology and parental perceptions of parent-child relationship quality. Recall that although an association between child internalizing problems and parent-perceived warmth and power assertion has been found (O'Brien \& Bahadur, 1998), a previous study examining a similar population found that child depressive symptomatology was not a significant predictor of parental perceptions of the parent-child relationship (Gerdes et al., 2003). It appears that the association between child depressive symptomatology and parental perceptions of relationship quality is not always straightforward and may depend on parental freedom from depression.

\section{Limitations}

Although the current study contributes to the literature in a number of ways, several limitations should be noted. As alluded to previously, the correlational nature of the present study does not allow us to determine causality. It is important to answer the question: Does experiencing symptoms of depression result in more negative child and parental perceptions of the relationship, or does having a poor parent-child relationship contribute to and maintain child and parental depressive affect? Future work should attempt to answer this question by employing a longitudinal design. Additionally, the exclusive self-report nature of the current study could be questioned; however, this is a difficult issue to address because self-report is widely regarded as the best measure of internalizing problems and certainly of perceptions of relationship quality. Many would argue that one's own perceptions of a relationship are key to understanding how the relationship affects them psychologically, regardless of what external sources or observers may report about the relationship. In addition, ADHD families included in the current study likely represent a select sample of children with ADHD because these children met stringent diagnostic criteria for Combined Type ADHD, and their parents agreed to participate in a longitudinal study and accept either psychosocial treatment or medication for their children. It is possible these findings may not generalize to other samples of children with ADHD. Finally, the measures examined in the current study were administered to ADHD families 10 months after treatment ended, which was the third or fourth time the measures had been administered to this group. Research on symptom attenuation suggests that reports of clinical symptoms decrease with repeated measurement (Jensen et al., 1995), which may have affected the results. However, such an effect would tend to erode the differences between the ADHD and LNCG groups, not spuriously create them. Nonetheless, future studies should attempt to replicate the current findings with simultaneously recruited samples of children with and without ADHD to control for possible symptom attenuation. 


\section{Clinical Implications}

Parent-child relationships play a major role in the development and adjustment of children. Understanding factors that contribute to child and parental perceptions of parent-child relationship quality has important clinical implications. If clinicians know what factors put families at risk for developing and maintaining negative parent-child relationships, they will be better equipped to intervene. The findings from the current study suggest that families with a depressive parent and children with either ADHD or depressive symptomatology may be particularly at risk for the development of a parent-child relationship characterized by low warmth and high power assertion. All three factors (child ADHD status, child depressive symptomatology, and parental depressive symptomatology) are amenable to proven effective interventions (Clarke, Rohde, Lewinsohn, Hops, \& Seeley, 1999; Gloaguen, Cottraux, Cucherat, \& Blackburn, 1998; Pelham \& Gnagy, 1999), suggesting important public health opportunities. By directly targeting ADHD and parental and child depression, clinicians will likely indirectly improve perceptions of the parent-child relationship, as well as change actual parenting behavior to that which is characterized by more warmth and less power assertion. A change in both perceptions and actual behavior is likely important because more positive child outcomes are associated with higher parental warmth and lower power assertion (e.g., O'Brien \& Bahadur, 1998), and perceptions of one's relationship are likely just as important, if not more important, than actual behavior in determining the psychological effect the relationship has on the parent and child.

\section{References}

Aiken, L. S., \& West, S. G. (1991). Multiple regression: Testing and interpreting interactions. Newbury Park, CA: Sage.

Arnold, L. E., Abikoff, H. B., Cantwell, D. P., Conners, C. K., Elliott, G., Greenhill, L. L., et al. (1997). National Institute of Mental Health collaborative Multimodal Treatment Study of Children with ADHD (the MTA): Design challenges and choices. Archives of General Psychiatry, 54, 865-870.

Asarnow, J. R., \& Bates, S. (1988). Depressive symptomatology in child psychiatric inpatients: Cognitive and attributional patterns. Journal of Abnormal Child Psychology, 16, 601-615.

Beck, A. T., Rush, A. J., Shaw, B. F., \& Emery, G. (1979). Cognitive therapy of depression. New York: Guilford Press.

Beck, A. T., Steer, R. A., \& Garbin, M. G. (1988). Psychometric properties of the Beck depression inventory: Twenty-five years of evaluation. Clinical Psychology Review, 8, 77-100.

Buhrmester, D., Camparo, L., Christensen, A., Gonzalez, L. S., \& Hinshaw, S. P. (1992). Mothers and fathers interacting in dyads and triads with normal and hyperactive sons. Developmental Psychology, 28, 500-509.

Campbell, S. B., March, C. L., Pierce, E. W., Ewing, L. J., \& Szumowski, E. K. (1991). Hard-to-manage preschool boys: Family context and the stability of externalizing behavior. Journal of Abnormal Child Psychology, 19, 301-318.

Clarke, G. N., Rohde, P., Lewinsohn, P. M., Hops, H., \& Seeley, J. R. (1999). Cognitive-behavioral treatment of adolescent depression: Efficacy of acute group treatment and booster sessions. Journal of the American Academy of Child and Adolescent Psychiatry, 38, 272-279.

Cummings, E. M., Keller, P. S., \& Davies, P. T. (2005). Towards a family process model of maternal and paternal depressive symptoms: Exploring multiple relations with child and family functioning. Journal of Child Psychology \& Psychiatry, 46, 479-489.

Diener, M. B., \& Milich, R. (1997). Effects of positive feedback on the social interactions of boys with Attention Deficit Hyper-activity Disorder: A test of the self-protective hypothesis. Journal of Clinical Child Psychology, 26, 256-265.

Downey, G., \& Coyne, J. C. (1990). Children of depressed parents: An integrative review. Psychological Bulletin, 108, 50-76.

Epkins, C. C. (1996). Affective confounding in social anxiety and dysphoria in children: Child, mother, and father reports of internalizing behaviors, social problems, and competence domains. Journal of Social and Clinical 
Psychology, 15, 449-470.

Furman, W., \& Giberson, R. S. (1995). Identifying the links between parents and their children's sibling relationships. In S. Shulman (Ed.), Close relationships in social-emotional development (pp. 95-108). Norwood, NJ: Ablex.

Gerdes, A. C., Hoza, B., \& Pelham, W. E. (2003). Attention-deficit/ hyperactivity disordered boys' relationships with their mothers and fathers: Child, mother, and father perceptions. Development and Psychopathology, 15, 363-382.

Gloaguen, V., Cottraux, J. Cucherat, M., \& Blackburn, I. M. (1998). A meta-analysis of the effects of cognitive therapy in depressed patients. Journal of Affective Disorders, 49, 59-72.

Greenhill, L. L., Abikoff, H. B., Arnold, L. E., Cantwell, D. P., Conners, C. K., Elliott, G., et al. (1996). Medication treatment strategies in the MTA study: Relevance to clinicians and researchers. Journal of the American Academy of Child \& Adolescent Psychiatry, 35, 1304-1313.

Herman, M. A., \& McHale, S. M. (1993). Coping with parental negativity: Links with parental warmth and child adjustment. Journal of Applied Developmental Psychology, 14, 121-136.

Hinshaw, S. P., \& Melnick, S. M. (1995). Peer relationships in boys with Attention-Deficit Hyperactivity Disorder with and without comorbid aggression. Development and Psychopathology, 7, 627-647.

Hoza, B., Gerdes, A. C., Hinshaw, S. P., Arnold, L. E., Pelham, W. E., Molina, B. S. G. et al. (2004). Self-perceptions of competence in children with ADHD and comparison children. Journal of Consulting \& Clinical Psychology, 72, 382-391.

Hoza, B., Pelham, W. E., Dobbs, J., Owens, J. S., \& Pillow, D. R. (2002). Do boys with Attention-Deficit/Hyperactivity Disorder have positive illusory self-concepts? Journal of Abnormal Psychology, 111, 268-278.

Hoza, B., Waschbusch, D. A., Pelham, W. E., Molina, B. S. G., \& Milich, R. (2000). ADHD and control boys' responses to social success and failure. Child Development, 71, 432-446.

Jensen, P. S., Roper, M., Fisher, P., Piacentini, J., Canino, G., Richters, J. et al. (1995). Test-retest reliability of the diagnostic interview schedule for children (DISC 2.1): Parent, child, and combined algorithms. Archives of General Psychiatry, 52, 61-71.

Johnston, C. (1996). Parent characteristics and parent-child interactions in families of nonproblem children and ADHD children with higher and lower levels of oppositional-defiant behavior. Journal of Abnormal Child Psychology, 24, 85-104.

Kovacs, M. (1992). Children's Depression Inventory (CDI) manual. North Tonawanda, NY: Multi-Health Systems.

McGrath, E. P., \& Repetti, R. L. (2002). A longitudinal study of children's depressive symptoms, self-perceptions, and cognitive distortions about the self. Journal of Abnormal Psychology, 111, 77-87.

MTA Cooperative Group. (1999). A 14-month randomized clinical trial of treatment strategies for Attention-Deficit/Hyperactivity Disorder. Archives of General Psychiatry, 56, 1073-1086.

O'Brien, M., \& Bahadur, M. A. (1998). Marital aggression, mother's problem-solving behavior with children, and children's emotional and behavioral problems. Journal of Social and Clinical Psychology, 17, 249-272.

Patterson, G. R. (1982). Coercive family process. Eugene, OR: Castalia.

Paulson, S. E., Hill, J. P., \& Holmbeck, G. N. (1991). Distinguishing between perceived closeness and parental warmth in families with seventh-grade boys and girls. Journal of Early Adolescence, 11, 276-293.

Pelham, W. E., \& Gnagy, E. M. (1999). Psychosocial and combined treatments for ADHD. Mental Retardation \& Developmental Disabilities Research Reviews, 5, 225-236.

Swanson, J. M. (1992). School-based assessments and interventions for ADD students. Irvine, CA: K. C. Publications.

Wells, K. C., Pelham, W. E., Kotkin, R. A., Hoza, B., Abikoff, H. B., Abramowitz, A., et al. (2000). Psychosocial treatment strategies in the MTA study: Rationale, methods, and critical issues in design and implementation. Journal of Abnormal Child Psychology, 28, 483-505. 